\title{
Intramanual and intermanual transfer of the curvature aftereffect
}

\author{
Bernard J. van der Horst • Maarten J. A. Duijndam • \\ Myrna F. M. Ketels · Martine T. J. M. Wilbers • \\ Sandra A. Zwijsen • Astrid M. L. Kappers
}

Received: 3 January 2008 / Accepted: 14 April 2008 / Published online: 26 April 2008

(C) The Author(s) 2008

\begin{abstract}
The existence and transfer of a haptic curvature aftereffect was investigated to obtain a greater insight into neural representation of shape. The haptic curvature aftereffect is the phenomenon whereby a flat surface is judged concave if the preceding touched stimulus was convex and vice versa. Single fingers were used to touch the subsequently presented stimuli. A substantial aftereffect was found when the adaptation surface and the test surface were touched by the same finger. Furthermore, a partial, but significant transfer of the aftereffect was demonstrated between fingers of the same hand and between fingers of both the hands. These results provide evidence that curvature information is not only represented at a level that is directly connected to the mechanoreceptors of individual fingers but is also represented at a stage in the somatosensory cortex shared by the fingers of both the hands.
\end{abstract}

Keywords Aftereffect - Curvature · Mechanoreceptor · Somatosensory $\cdot$ Tactile $\cdot$ Touch $\cdot$ Transfer

\section{Introduction}

The neural representation of haptic information can be investigated using different approaches. The representation of object shape perceived with the fingers has mainly been studied using neurophysiological tools. It has been found that especially slowly adapting type I (SAI) mechanoreceptors

B. J. van der Horst $(\bowtie) \cdot$ M. J. A. Duijndam · M. F. M. Ketels · M. T. J. M. Wilbers · S. A. Zwijsen - A. M. L. Kappers

Department of Physics of Man, Helmholtz Instituut,

Universiteit Utrecht, Princetonplein 5,

3584 CC Utrecht, The Netherlands

e-mail: b.j.vanderhorst@uu.nl in the finger but also fast-adapting type I (FAI) receptors are sensitive to curvature (Goodwin et al. 1997; Jenmalm et al. 2003). In order to perceive curvature, a combination of responses from a population of receptors is required (Goodwin and Wheat 2004). This processing occurs along several stages up to at least the somatosensory cortex (SI) (Gardner and Kandel 2000). Taking a neurophysiological approach is useful to uncover the pathways underlying curvature processing, but is less appropriate to establish the levels at which perceived curvature is essentially represented.

A psychophysical approach that has been successful in providing greater insight into the neural representation of perceived properties is the study of the aftereffect, and especially, the transfer of the aftereffect. In vision, for example, the finding of partial, interocular transfer of the motion aftereffect has been explained by the involvement of both monocular and binocular cells in the processing of motion information from the stimulus (Moulden 1980; Wade et al. 1993; Tao et al. 2003). In a similar way, establishing the transfer characteristics of a haptic curvature aftereffect would provide insight into the representation of shape information. Finding aftereffect transfer between different fingers would indicate that curvature is represented at a level shared by these fingers, whereas no transfer would imply that each finger has a separate representation of curvature.

A curvature aftereffect is the phenomenon whereby a flat test surface feels concave following prolonged contact with a convex adaptation surface (see Fig. 1a). Curvature aftereffects have been found for different shapes and exploration modes. Gibson (1933) reported that a flat cardboard edge felt concave after the prolonged dynamic exploration of a convex cardboard edge. Vogels et al. (1996) demonstrated the existence of an aftereffect when the whole hand was placed on spherically curved shapes. They performed 


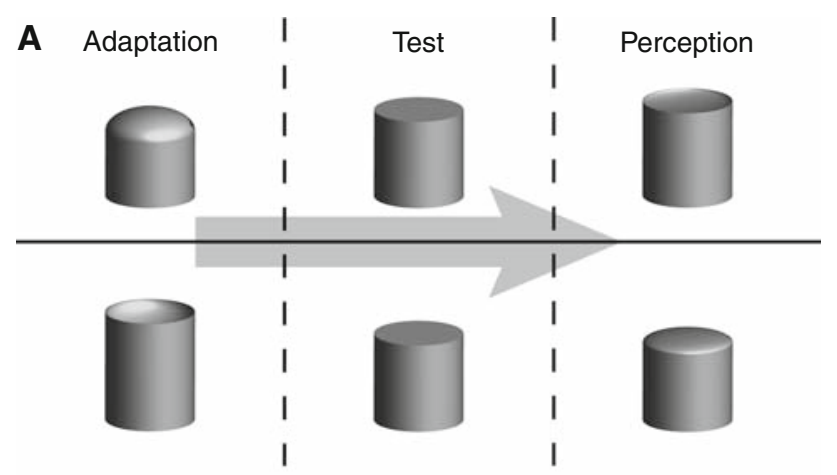

B
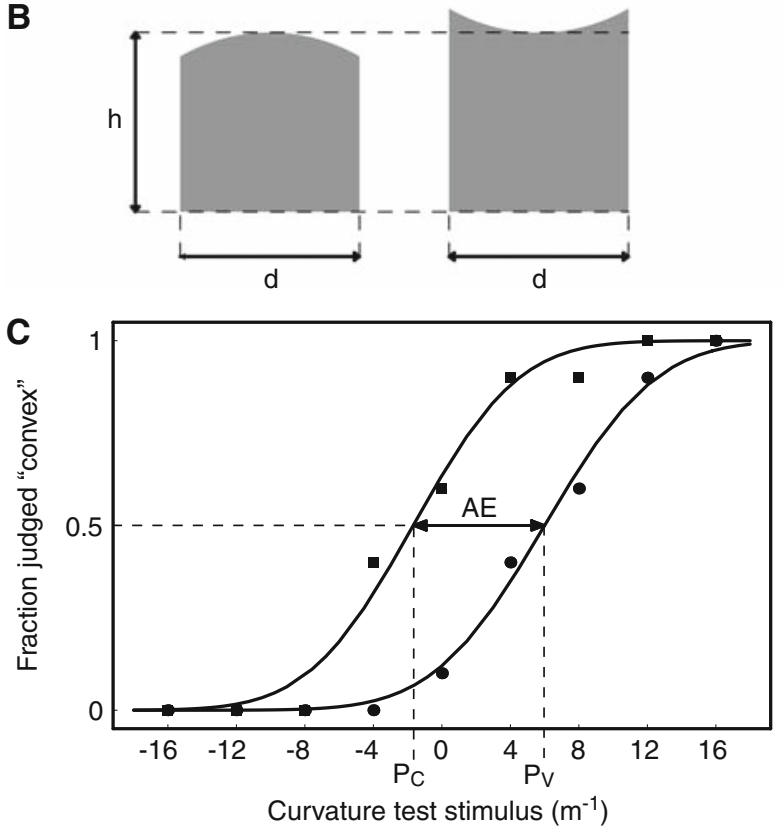

Fig. 1 a Schematic overview of a haptic curvature aftereffect: when you first touch a convex (concave) surface for some time, say $10 \mathrm{~s}$, and subsequently touch a flat surface, this latter surface feels concave (convex). b Schematic drawings of the cross-sections of a convex and a concave stimulus. The stimuli had a cylindrical shape with a spherical top (see illustration a). The distance from the bottom to the centre of the top $(h)$ was consistently $30 \mathrm{~mm}$. The diameter of the cylinders $(d)$ was also $30 \mathrm{~mm}$. c Examples of two psychometric curves. The circular data points and the fit through these points results from adaptation to the convex adaptation stimulus. The PSE is represented by $P_{\mathrm{V}}$. The square data points and the fit through these points result from adaptation to the concave adaptation stimulus. In this case, the PSE is represented by $P_{\mathrm{C}}$. The magnitude of the aftereffect (AE) is defined as the difference between $P_{\mathrm{V}}$ and $P_{\mathrm{C}}$

extensive experiments to examine the characteristics of this static curvature aftereffect. They found a linear relationship between the magnitude of the aftereffect and the curvature of the adaptation stimulus. Furthermore, they showed that the magnitude of the aftereffect increased with the adaptation time up to about $10 \mathrm{~s}$. Finally, they found a decrease of the aftereffect with an increase of the interstimulus interval. In a follow-up study, they showed that the aftereffect also existed for alternative exploration modes, like touching a stimulus with only the five fingertips of the hand or performing small movements of the hand over the stimulus surface (Vogels et al. 1997). Given the strength and consistency of these findings, we supposed that curvature aftereffects should also occur for alternative ways of touching, such as the situation in which curved surfaces are statically being touched with only a single fingertip. However, this phenomenon has not yet been investigated, and consequently, any curvature aftereffect transfer between the fingers also remains unexplored.

The purpose of the present study was to obtain a better understanding of the representation of haptically perceived shape information, by probing the transfer of the curvature aftereffect. In the first experiment, we established the existence of an aftereffect when a curved surface is touched by a single finger and measured whether this aftereffect transferred to other fingers of the same hand. The second experiment was set up to determine whether the aftereffect depended on the finger used. Finally, in the third and fourth experiments, we investigated the transfer of the aftereffect between fingers of both hands.

\section{Materials and methods}

\section{Subjects}

A total number of 40 subjects participated $[n=8$ for experiments 1,2 and $4, n=16$ for experiment $3 ; 18$ were male and 22 were female; the mean age was 22 years; 37 were right-handed, 3 were left-handed, according to a standard questionnaire (Coren 1993)]. Subjects in experiments 1 and 2 received course credit for their participation. Subjects in the third and fourth experiments received monetary compensation.

\section{Stimuli}

The stimuli comprised of a compound of polyurethane foam and artificial resin (Cibatool BM 5460). A computercontrolled milling machine was used to produce cylinders with a flat bottom and a spherically curved top. The top was either pointing outward (convex) or inward (concave). Both convex and concave adaptation stimuli were used, with curvature values of +36 and $-36 \mathrm{~m}^{-1}$, respectively; the curvature of the nine test stimuli ranged from -16 to $+16 \mathrm{~m}^{-1}$, in steps of $4 \mathrm{~m}^{-1}$. Illustrations of the stimuli and their crosssections are given in Fig. 1a, b, respectively.

\section{Procedure}

A subject was seated behind a table. The preferred arm rested on a platform, which was $30 \mathrm{~mm}$ above the tabletop. 
In the third and fourth experiments, both arms rested on the platform. Only the fingertips projected over the platform. The experimenter placed the stimulus underneath a fingertip. A curtain prevented the subjects from seeing the stimulus. During a trial, the tip of one finger was placed on an adaptation stimulus for $10 \mathrm{~s}$. Subsequently, the subject placed a finger on a test stimulus and had to judge whether this test stimulus felt convex or concave. Subjects were not allowed to move the finger over the stimulus surface, and the experimenter checked for this. No instructions were given on the force to contact the stimulus, nor was it measured. No feedback was provided on the response.

Three conditions were measured in the first experiment. In all conditions, the adaptation stimulus was touched with the index finger. In one condition, the test stimulus was also touched with the index finger. In the other two conditions, the test stimulus was touched with the middle finger or the little finger of the same hand, respectively. Each condition consisted of 10 repetitions of a group of 18 trials (two adaptation stimuli $\times$ nine test stimuli) with trials randomized within a group. One complete condition was measured in a single session of about one and a half hours. The separate sessions were spread over different days. The order in which the conditions were conducted was counterbalanced for the first six subjects and randomly chosen for the last two subjects.

In the second experiment, both the adaptation and the test stimuli were touched by the middle finger. In the third and fourth experiments, the adaptation stimulus was contacted by the index finger of the preferred hand; the test stimuli were touched with the index finger (third experiment) or middle finger (fourth experiment) of the non-preferred hand.
Analysis

The data for each subject and each condition were analyzed separately for the convex and the concave adaptation stimuli. The percentage of "convex" responses was plotted against the curvature of the test stimulus. The point of subjective equality (PSE) was determined by fitting a psychometric function (cumulative Gaussian) to the data. The PSE represents the curvature value that in $50 \%$ of the test cases was judged "convex" and in 50\% of the cases was judged "concave". The magnitude of the aftereffect is defined as the difference between the PSE resulting from the adaptation to a convex surface and the PSE resulting from the adaptation to a concave surface. Examples of psychometric curves for a convex and a concave adaptation are given in Fig. 1c.

\section{Results}

The mean results for the aftereffect values are shown in Fig. 2. The error bars indicate the standard errors of the mean.

\section{Experiment 1}

We tested the occurrence of an aftereffect in each condition by performing separate one-tailed $t$ tests. A significant result was obtained in all conditions $\left(t_{7}=6.3, P<0.001\right.$ for the index finger; $t_{7}=9.8, P<0.001$ for the middle finger; $t_{7}=3.4, P=0.006$ for the little finger). Subsequently, an ANOVA with a repeated measures design was performed

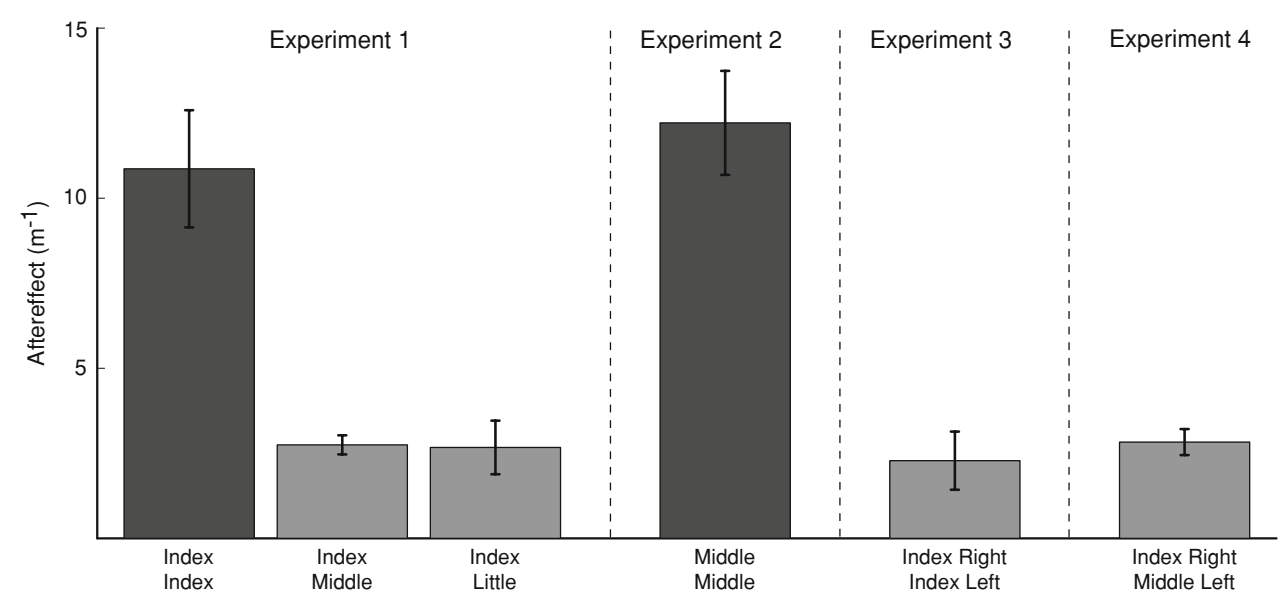

Fig. 2 Mean results of the aftereffect. The indicated error bars are the standard error in the mean for each condition. Experiment 1: eight subjects participated. Adaptation was performed by the index finger of the preferred hand. Testing was done using the index finger, middle finger, or little finger of the same hand. Experiment 2: eight subjects participated. Adaptation and testing was performed by the middle finger of

the preferred hand. Experiment 3: sixteen subjects participated. Adaptation was performed by the index finger of the preferred hand; testing was done by the opposite index finger. Experiment 4: eight subjects participated. Adaptation was performed by the index finger of the preferred hand; testing was done by the middle finger of the non-preferred hand 
to determine any differences between conditions. A significant main effect was found $\left(F_{2,14}=22.5, P<0.001\right)$. Pairwise comparisons showed a significant difference between the index finger and the middle finger $(P=0.007)$ and between the index finger and the little finger $(P=0.004)$, but not between the middle finger and the little finger $(P=1.0)$. The $P$ values were adjusted with a Bonferroni correction.

\section{Experiment 2}

A one-tailed $t$ test showed that there was a significant aftereffect $\left(t_{7}=8.0, P<0.001\right)$. Inspection of Fig. 2 shows that the aftereffect of the middle finger condition of the second experiment was comparable to the index finger condition of the first experiment and was much higher than the middle finger condition of the first experiment. Independent samples $t$ test confirmed that there was no significant difference in the first case $\left(t_{14}=0.6, P=0.6\right)$, but that there was a significant difference in the second case $\left(t_{7.4}=6.1, P<0.001\right)$.

\section{Experiment 3}

A one-tailed $t$ test highlighted a significant aftereffect $\left(t_{15}=2.7, P=0.009\right)$. The magnitude of this aftereffect was much lower than for the index finger condition of the first experiment. This was confirmed by an independent sample $t$ test $\left(t_{22}=5.0, P<0.001\right)$.

\section{Experiment 4}

A significant aftereffect was obtained, as was confirmed by a one-tailed $t$ test $\left(t_{7}=7.4, P<0.001\right)$.

\section{Discussion}

The first novel observation of this paper is that the perception of surface curvature by a single fingertip is influenced by preceding contact of this finger with another curved surface. The magnitude of this curvature aftereffect did not depend on the finger employed, as shown by a comparison between the results of the first and the second experiment. Previously, Vogels et al. $(1996,1997)$ reported the existence of a static curvature aftereffect, when the whole hand was used. We suppose that our finding of a one-finger aftereffect falls in the same class of phenomena. A quantitative comparison between the results of Vogels et al. (1996) and our finding can be made by calculating the relative magnitude of the aftereffect, i.e. the aftereffect divided by the difference between the adaptation stimuli. This value equals $0.17 \pm 0.02$ for the results of Vogels et al., whereas it was $0.15 \pm 0.07$ for the index finger condition of the first exper- iment and $0.17 \pm 0.06$ for the middle finger condition of the second experiment, respectively. These values are in the same order of magnitude, irrespective of the differences in manner of touching and curvature range of the stimuli.

The second important finding of our study is that the aftereffect partially transfers between fingers of the same hand. This means that the sensation of shape with a certain finger influences the perception of a shape touched by another finger. This suggests that the sensations obtained by the different fingers share a common representation. However, the transfer is far from complete, indicating that curvature perception by each finger also yields a substantial, individual part in the representation. Interestingly, the aftereffect does not only transfer from the index finger to the neighboring middle finger, but also to the distant little finger. This result is unlike recently performed localization (Schweizer et al. 2000) and learning studies (Sathian and Zangaladze 1997; Harris et al. 2001), in which the reported transfer effects were obtained in the neighboring finger, but not in the distant fingers. This indicates that the processes involved in detecting the finger that is stimulated or increasing the skills to discriminate punctate pressure or roughness are quite different from those concerned in shape perception of an object.

The third interesting result of this study is that there was a small, but significant transfer of the aftereffect between fingers of both hands, irrespective of whether opposite fingers (experiment 3) or different fingers (experiment 4) were employed. This result is different from the result reported by Vogels et al. 1997, who did not find intermanual transfer. However, in their experiments, whole hands were involved, whereas only single fingertips were used in our experiment. Moreover, their conclusion was based on the performance of only 2 subjects, whereas 24 participants provided the data for our study. The results of the third and fourth experiments suggest that the representation of shape information obtained with one hand is not completely distinct from the representation of shape information received by the other hand, but shares a common, bilateral component.

How can our findings be interpreted in the context of neurophysiological literature? Firstly, our finding that the aftereffect only transfers partially between fingers of the same hand shows that a substantial part of the processing occurs at a stage where each finger is individually represented. On this stage, which spreads from the mechanoreceptors in the fingers up to area $3 \mathrm{~b}$ in SI, no overlap occurs in signals from the slowly adapting receptors and the fastadapting receptors (Gardner and Kandel 2000). Slowly adapting receptors respond with a sustained discharge when the finger is in contact with a surface, whereas fast-adapting receptors only respond at the onset and removal phase of the finger (Johansson and Vallbo 1983). Vogels et al. 
(1996) showed that the magnitude of their curvature aftereffect increased with an increase in adaptation time. These findings point to an important role for the slowly adapting receptors in the curvature aftereffect. Therefore, we suggest that the aftereffect at the stage related to an individual finger mainly originates from the processing of the slowly adapting receptors. Secondly, the fact that we found a transfer between the fingers of the same hand implies that a significant part of the processing of curvature information occurs at a level shared by the different fingers. In physiological terms, this indicates that at least area 1 or 2 of SI are involved, as receptive fields in these areas cover several fingers of a single hand (Gardner and Kandel 2000), but processing may also occur at an even higher stage. Thirdly, our finding of an intermanual transfer shows that the processing of curvature information also takes place on a higher, bilateral level. We can only speculate on the neural correlates of this bilateral processing. Possible candidates include area 2 of SI, areas 5 and 7 of the posterior parietal cortex, and the secondary somatosensory cortex (Iwamura 2000; Gardner and Kandel 2000).

It is interesting to mention that the aftereffects in the intramanual transfer conditions (experiment 1) and the intermanual transfer conditions (experiment 3 and 4) are similar in magnitude. This suggests that no important curvature processing occurs at a level that is devoted to a single hand, but that all processing takes place at a higher stage. The similar results for experiments 3 and 4 provide further support that the hands and fingers are not somatotopically represented at this stage. From a previous study, it is known that subjects also performed similarly in intramanual and intermanual curvature discrimination tasks, but that higher performance was obtained when only a single finger was employed (Van der Horst and Kappers 2007). The analogy between that study and the current study is that curvature information is mainly represented at the level of the individual finger, but partly available at a higher, fingerand hand-independent level. We should be careful in ascribing a specific function to the involvement of the higher level areas in the processing of curvature information. The role of more cognitive aspects should not be excluded, since it is known that processes like tactile attention (Burton and Sinclair 2000; Spence and Gallace 2007), working memory (Burton and Sinclair 2000), and object recognition (Reed et al. 2004) also engage the somatosensory areas.

The aftereffect that we found in the present study is a similar phenomenon as the aftereffect that was previously reported by Vogels et al. (1996, 1997). However, this does not entail that the representation of curvature is identical for touching with a single finger or with the whole hand. Vogels et al. (1997) already showed that, although similar aftereffects were found when either the whole hand or only the five fingertips were used, only a small transfer between these exploration modes was obtained, which points to a limited overlap in representation. Similarly, we suppose that there is a difference in representation between curvature that is perceived by a single finger and curvature that is perceived by the whole hand. In the single finger case, the representation is mainly at the level of the individual finger, whereas in the whole hand case, the representation is spread over all fingers and the palm of the hand.

This study shows that establishing the intramanual and intermanual transfer of the aftereffect is a useful tool in obtaining more insight into the representation of object properties as perceived by the fingers. In general, studying aftereffect transfer is attractive, because it enables a connection between psychophysics and neurophysiology. The convergence of these approaches leads to a better understanding of human perception.

Acknowledgment This research was supported by a grant from the Netherlands Organisation for Scientific Research (NWO).

Open Access This article is distributed under the terms of the Creative Commons Attribution Noncommercial License which permits any noncommercial use, distribution, and reproduction in any medium, provided the original author(s) and source are credited.

\section{References}

Burton H, Sinclair RJ (2000) Attending to and remembering tactile stimuli: a review of brain imaging data and single-neuron responses. J Clin Neurophysiol 17:575-591

Coren S (1993) The left-hander syndrome. Vintage Books, New York

Gardner EP, Kandel ER (2000) Touch. In: Kandel ER, Schwartz JH, Jessell TM (eds) Principles of neural science, 4th edn. McGrawHill, New York, pp 451-471

Gibson JJ (1933) Adaptation, after-effect and contrast in the perception of curved lines. J Exp Psychol 16:1-31

Goodwin AW, Macefield VG, Bisley JW (1997) Encoding of object curvature by tactile afferents from human fingers. J Neurophysiol 78:2881-2888

Goodwin AW, Wheat HE (2004) Sensory signals in neural populations underlying tactile perception and manipulation. Annu Rev Neurosci 27:53-77

Harris JA, Harris IM, Diamond ME (2001) The topography of tactile learning in humans. J Neurosci 21:1056-1061

Iwamura Y (2000) Bilateral receptive field neurons and callosal connections in the somatosensory cortex. Philos Trans R Soc Lond B Biol Sci 355:267-273

Jenmalm P, Birznieks I, Goodwin AW, Johansson RS (2003) Influence of object shape on responses of human tactile afferents under conditions characteristic of manipulation. Eur J Neurosci 18:164 176

Johansson RS, Vallbo ÅB (1983) Tactile sensory coding in the glabrous skin of the human hand. Trends Neurosci 6:27-32

Moulden B (1980) After-effects and the integration of patterns of neural activity within a channel. Philos Trans R Soc Lond B Biol Sci 290:39-55

Reed CL, Shoham S, Halgren E (2004) Neural substrates of tactile object recognition: an fMRI study. Hum Brain Mapp 21:236246 
Sathian K, Zangaladze A (1997) Tactile learning is task specific but transfers between fingers. Percept Psychophys 59:119-128

Schweizer R, Maier M, Braun C, Birbaumer N (2000) Distribution of mislocalizations of tactile stimuli on the fingers of the human hand. Somatosens Mot Res 17:309-316

Spence C, Gallace A (2007) Recent developments in the study of tactile attention. Can J Exp Psychol 61:196-207

Tao R, Lankheet MJM, van de Grind WA, van Wezel RJA (2003) Velocity dependence of the interocular transfer of dynamic motion aftereffects. Perception 32:855-866
Van der Horst BJ, Kappers AML (2007) Curvature discrimination in various finger conditions. Exp Brain Res 177:304-311

Vogels IMLC, Kappers AML, Koenderink JJ (1996) Haptic aftereffect of curved surfaces. Perception 25:109-119

Vogels IMLC, Kappers AML, Koenderink JJ (1997) Investigation into the origin of the haptic after-effect of curved surfaces. Perception 26:101-117

Wade NJ, Swanston MT, de Weert CMM (1993) On interocular transfer of motion aftereffects. Perception 22:1365-1380 